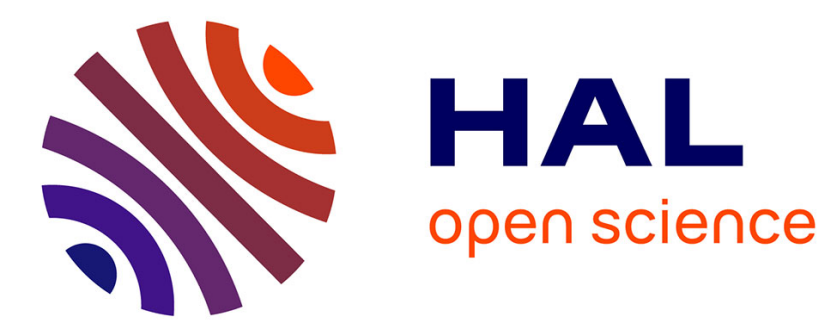

\title{
Equidispersive Dual-Mode Long Slot Arrays
}

M. del Mastro, S. Lenzini, R. Sauleau, Mauro Ettorre

\section{To cite this version:}

M. del Mastro, S. Lenzini, R. Sauleau, Mauro Ettorre. Equidispersive Dual-Mode Long Slot Arrays. IEEE Antennas and Wireless Propagation Letters, 2020, 19 (12), pp.2127-2131. 10.1109/LAWP.2020.3024618 . hal-03103351

\section{HAL Id: hal-03103351 \\ https://hal.science/hal-03103351}

Submitted on 11 Jan 2021

HAL is a multi-disciplinary open access archive for the deposit and dissemination of scientific research documents, whether they are published or not. The documents may come from teaching and research institutions in France or abroad, or from public or private research centers.
L'archive ouverte pluridisciplinaire HAL, est destinée au dépôt et à la diffusion de documents scientifiques de niveau recherche, publiés ou non, émanant des établissements d'enseignement et de recherche français ou étrangers, des laboratoires publics ou privés. 


\title{
Equi-Dispersive Dual-Mode Long Slot Arrays
}

\author{
Michele Del Mastro, Stefano Lenzini, Ronan Sauleau, Fellow, IEEE, and Mauro Ettorre, Senior Member, IEEE
}

\begin{abstract}
In this letter, we propose an analytic study for engineering equi-dispersive (ED) ortho-mode parallel plate waveguide (PPW) structures. As difference with classical PPWs having perfectly conducting walls, the metallic plates are here replaced by arbitrary surface impedance boundaries. Closed-form expressions are provided to engineer their dispersion and obtain purely ED dual-mode propagation exploiting transverse electric and magnetic modes. This new class of PPWs is finally used as radiating open-ended stubs, arranged in a periodic environment to attain circular polarization. The corresponding axial ratio is calculated to be below $3 \mathrm{~dB}$ over a $22.3 \%$ fractional bandwidth.
\end{abstract}

Index Terms-equi-dispersive, ortho-mode, parallel plate waveguide, circular polarization, impedance boundary condition, continuous transverse stub array.

\section{INTRODUCTION}

$\mathbf{M}$ ICROWAVE components, supporting orthogonallypolarized degenerate modal branches, are widely used to generate circular or dual-linear polarization. For instance, square/circular waveguides are used in septum polarizers [1], [2], or as unit cell of phased arrays attaining polarization agility as well as circular polarization (CP) [3], [4].

A different case-of-study is the parallel-plate waveguide (PPW), which supports degenerate transverse electric (TE) and magnetic (TM) modes. However, PPWs with perfect electric conductive plates have the drawback of supporting also a transverse electromagnetic (TEM) propagation, which represents an additional source of inter-modal dispersion. The use of dual-mode PPWs may result in innovative feeding and radiating structures. The articles [5]-[8] have recently proposed a novel concept of continuous transverse stub (CTS) arrays made of dual-mode PPWs, able to generate $\mathrm{CP}$ or duallinear polarization over a wide band and large field-of-view.

In this letter, we present an analytic study of PPWs with properly engineered pure reactive walls as a possible strategy to inhibit the TEM propagation and to reach the aimed equidispersive (ED), ortho-mode propagation. The study provides analytic expressions to analyze these structures. It is also shown that ED PPWs exhibit high CP purity, when used as radiating stubs.

The paper is organized as follows: Section II outlines the modal analysis of the problem. The dispersion engineering is exhaustively discussed in Sections III and IV. Section $\mathrm{V}$ presents the radiation of open-ended ED PPWs. Finally, Section VI concludes the letter.

Manuscript received XX/XX/2018; revised XX/XX/2018. "This work was supported by the Direction Générale de l'Armement and by Brittany Region, France.

M. Del Mastro, R. Sauleau and M. Ettorre are with Univ. Rennes, CNRS, IETR (Institut d'Électronique et des Technologies du numéRique) - UMR 6164, F-35000 Rennes, France (e-mail: michele.delmastro@univ-rennes1.fr).

S. Lenzini is with University of Modena and Reggio Emilia, Department of Engineering "Enzo Ferrari", 41125 Modena, Italy.

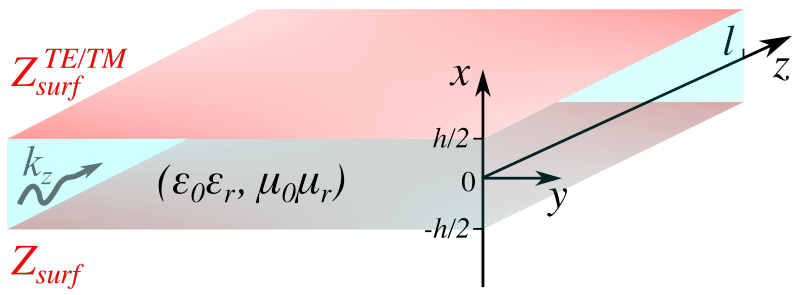

Fig. 1: Geometry of the problem under analysis. The structure consists of two parallel plates, infinitely-extended along $y$-axis and modeled as surface impedance with respect to TE and TM modes. Modes propagate along $z$-axis.

\section{Modal AnAlysis}

In the following, we will focus on a non-standard PPW as shown in Fig. 1. The bounding walls of the considered PPW are modeled using impedance boundary conditions (IBC). The structure is infinitely-extended along $y$-axis and the propagation is intended along $z$-axis. Given the absence of source, the problem reduces to solving the Helmholtz equation and searching for the corresponding eigenvalues [9]-[11]:

$$
\mathcal{H}\left\{\phi^{T E / T M}\right\}=0
$$

where $\phi^{T E / T M}$ is an arbitrary chosen scalar potential and $\mathcal{H}\{\cdot\}=\nabla_{t}^{2}+\left|k_{t}^{T E / T M}\right|^{2}=\partial_{x}^{2}+\partial_{y}^{2}+\left(k_{x}^{T E / T M}\right)^{2}+$ $\left(k_{y}^{T E / T M}\right)^{2}$ is the Helmholtz operator with $k_{x}^{T E / T M}$ and $k_{y}^{T E / T M}$ being the transverse eigenvalues for both TE and TM modes, accordingly. It can be demonstrated that $k_{y}^{T E / T M}=0$ by virtue of the infinite extension of the structure in Fig. 1 along $y$-axis [11]. The eigenvalues $k_{x}^{T E / T M}$ are found by enforcing the IBCs on the waveguide contour, yielding

$$
\mathbf{E}_{\mathbf{t}}^{\mathbf{T E} / \mathbf{T M}}=Z_{\text {surf }}^{T E / T M} \hat{n} \times \mathbf{H}^{\mathbf{T E} / \mathbf{T M}}
$$

where $\mathbf{E}_{\mathbf{t}}=E_{y} \hat{y}+E_{z} \hat{z}$ and $\hat{n}$ is the inward unit vector, normal to the parallel plates. Moreover, $Z_{\text {surf }}^{T E / T M}$ is the surface impedance for TE and TM modes delimiting the waveguide cross section. We will assume $Z_{\text {surf }}^{T E / T M}$ to be purely reactive hereinafter. Studying TE and TM modes independently, the two parallel plates exhibit identical surface impedance.

\section{A. TE modes in PPW with impedance walls}

A convenient choice for the scalar potential $\phi^{T E}$ is inspired by [12] and takes the form $\phi^{T E}=A^{T E}\left[\xi^{T E+}+B^{T E} \xi^{T E-}\right]$, where $\xi^{T E \pm}=e^{ \pm i k_{x}^{T E}(x+h / 2)}$ and $h$ is the height of the PPW. Also, $A^{T E}, B^{T E}$ are constants to be determined. For 
TE modes, the eigenvalues $k_{x}^{T E}$ are calculated enforcing the IBC (2) that reduces to the following form

$$
Z_{\text {surf }}^{T E}=\left.\frac{E_{y}^{T E}}{H_{z}^{T E}}\right|_{x=h / 2} \quad Z_{\text {surf }}^{T E}=-\left.\frac{E_{y}^{T E}}{H_{z}^{T E}}\right|_{x=-h / 2}
$$

By using the field expressions of hollow waveguides [9], the IBC (3) can be computed as function of the scalar potential $\phi^{T E}$, yielding to $B^{T E \pm}= \pm e^{i k_{x}^{T E \pm} h}$. Two separate field solutions are so found, which will be referred to using the superscripts \pm . Hence, the characteristic equation (CE) can be expressed as function of $Z_{\text {surf }}^{T E}$ and arranged as follows

$$
Z_{\text {surf }}^{T E \pm}=\frac{\omega \mu_{0} \mu_{r}}{k_{x}^{T E \pm}} \frac{1 \mp e^{i k_{x}^{T E \pm} h}}{1 \pm e^{i k_{x}^{T E \pm} h}}
$$

where $\omega$ is the angular frequency and $\mu_{r}$ is the dielectric permeability of the material filling the PPW. The obtained CE (4) is transcendental and must be numerically solved for the eigenvalues $k_{x}^{T E \pm}$. Since we are studying a closeddomain problem, a countable set of $k_{x}^{T E \pm}$ is obtained, i.e., $k_{x, m=1,2, \ldots,+\infty}^{T E \pm}$. In this paper, we will use a Padé-approximantbased root-finding procedure as presented in [13].

The field modal vectors are calculated using the scalar potential $\phi^{T E}$ and, after some algebraic manipulations, can be written as

$$
\begin{aligned}
& \left.\boldsymbol{e}_{\boldsymbol{t}}^{\boldsymbol{T E} \pm}=e_{y}^{T E \pm} \hat{y}=\begin{array}{c}
i A^{T E+} \sin \left(k_{x}^{T E+} x\right) \\
A^{T E-} \cos \left(k_{x}^{T E-} x\right)
\end{array}\right) \hat{y} \\
& \boldsymbol{h}_{\boldsymbol{z}}^{\boldsymbol{T E} \pm}=h_{z}^{T E \pm} \hat{z}=i \partial_{x} e_{y}^{T E \pm} /\left(\omega \mu_{0} \mu_{r}\right) \hat{z} \\
& \boldsymbol{h}_{\boldsymbol{t}}^{\boldsymbol{T E} \pm}=h_{x}^{T E \pm} \hat{x}=\hat{z} \times \boldsymbol{e}_{\boldsymbol{t}}^{\boldsymbol{T E} \pm}
\end{aligned}
$$

where

$$
A^{T E \pm}=\sqrt{2 / h}\left(\operatorname{sinc}\left(k_{x}^{T E \pm} h\right) \mp 1\right)^{-1 / 2}
$$

which is found by enforcing the orthonormality property of waveguide modes [9]. The propagation constant is given by $k_{z}^{T E \pm}=\sqrt{k_{0}^{2} \epsilon_{r} \mu_{r}-\left(k_{x}^{T E \pm}\right)^{2}}$, where $k_{0}$ is the free space wavenumber.

\section{B. TM modes in PPW with impedance walls}

The TM mode scenario is solved considering the following scalar potential $\phi^{T M}=A^{T M}\left[\xi^{T M+}+B^{T M} \xi^{T M-}\right]$ (where $\left.\xi^{T M \pm}=e^{ \pm i k_{x}^{T M}(x+h / 2)}\right)$ and enforcing the IBC given by

$$
Z_{\text {surf }}^{T M}=-\left.\frac{E_{z}^{T M}}{H_{y}^{T M}}\right|_{x=h / 2} \quad Z_{\text {surf }}^{T M}=\left.\frac{E_{z}^{T M}}{H_{y}^{T M}}\right|_{x=-h / 2}
$$

The corresponding $\mathrm{CE}$ is given by the following two-form relation

$$
Z_{\text {surf }}^{T M \pm}=\frac{k_{x}^{T M \pm}}{\omega \epsilon_{0} \epsilon_{r}} \frac{1 \pm e^{i k_{x}^{T M \pm} h}}{1 \mp e^{i k_{x}^{T M \pm} h}}
$$

where $\epsilon_{r}$ is the dielectric permittivity of the material filling the PPW. Solving (8), a discrete set of solutions is found, i.e., $k_{x, m=1,2, \ldots,+\infty}^{T M \pm}$. The TM modal vectors are given by

$$
\begin{aligned}
& \left.\boldsymbol{e}_{\boldsymbol{t}}^{\boldsymbol{T M}}=e_{x}^{T M \pm} \hat{x}=\begin{array}{c}
i A^{T M+} \sin \left(k_{x}^{T M+} x\right) \\
A^{T M-} \cos \left(k_{x}^{T M-} x\right)
\end{array}\right) \hat{x} \\
& \boldsymbol{h}_{\boldsymbol{t}}^{\boldsymbol{T M} \pm}=h_{y}^{T M \pm} \hat{y}=\hat{z} \times \boldsymbol{e}_{\boldsymbol{t}}^{\boldsymbol{T} M \pm} \\
& \boldsymbol{e}_{\boldsymbol{z}}^{\boldsymbol{T} M \pm}=e_{z}^{T M \pm} \hat{z}=-i \partial_{x} h_{y}^{T M \pm} /\left(\omega \epsilon_{0} \epsilon_{r}\right) \hat{z}
\end{aligned}
$$



Fig. 2: Impedance loci of TE and TM modes when the PPW's height is $h=\lambda / 2$, where $\lambda$ is the wavelength at $40 \mathrm{GHz}$. The characteristic equations are plotted at the operating frequency. The PPW is air-filled.

where

$$
A^{T M \pm}=\sqrt{2 / h}\left(\operatorname{sinc}\left(k_{x}^{T M \pm} h\right) \mp 1\right)^{-1 / 2}
$$

Finally, the propagation constant can be written as $k_{z}^{T M \pm}=$ $\sqrt{k_{0}^{2} \epsilon_{r} \mu_{r}-\left(k_{x}^{T M \pm}\right)^{2}}$.

\section{EQUi-DISPERSIVE ORTHO-MOde PPW}

Unlike standard PPW, the proposed type of waveguide can support two orthgonally-polarized modes having same dispersive behavior. This valuable property is observed by linking their propagation to the required surface impedance (4) and (8), as shown in Fig. 2. Specifically, if the PPW's height is less than a half of the operating wavelength $(h \leq \lambda / 2)$ and the surface impedance is capacitive for TM modes and inductive for TE ones, the set of solutions for (4) and (8) has only two eigenvalues $k_{x}^{T E}$ and $k_{x}^{T M}$ in the visible range $\left(\left|k_{x}^{T E / T M}\right|<k_{0} \sqrt{\epsilon_{r} \mu_{r}}\right)$, respectively. Note that this is true only in case $Z_{\text {surf }}^{T M}$ is capacitive and $Z_{\text {surf }}^{T E}$ is inductive. In more general scenarios, surface-wave modes $\left(k_{x}^{T E}=\Im\left\{k_{x}^{T E}\right\}\right.$ and $k_{x}^{T M}=\Im\left\{k_{x}^{T M}\right\}$ ) may be present too [14].

The ED condition is achieved by engineering the surface impedance $Z_{\text {surf }}^{T M / T E}$. Let us model $Z_{\text {surf }}^{T M}$ and $Z_{\text {surf }}^{T E}$ as being purely capacitive and inductive, respectively:

$$
\begin{gathered}
Z_{\text {surf }}^{T E-}=i \omega L^{T E} \\
Z_{\text {surf }}^{T M-}=\left(i \omega C^{T M}\right)^{-1}
\end{gathered}
$$

The ED condition can be found by inserting (11) into (4) and (12) into (8) and enforcing $k_{x}^{T E-}=k_{x}^{T M-}$. The product $Z_{\text {surf }}^{T E-} Z_{\text {surf }}^{T M-}$ yields to the following ED condition:

$$
L^{T E}=C^{T M} \eta^{2}
$$

where $\eta=\eta_{0} \sqrt{\mu_{r} / \epsilon_{r}}$ and $\eta_{0}=120 \pi \Omega$. The capacitance $C^{T M}$ is calculated by arbitrarily setting the cut-off frequency $f_{c o}^{T M}$ of the TM mode, yielding

$$
C^{T M}=\left(\omega_{c o}^{T M} \eta\right)^{-1} \cot (k h / 2)
$$






(a)

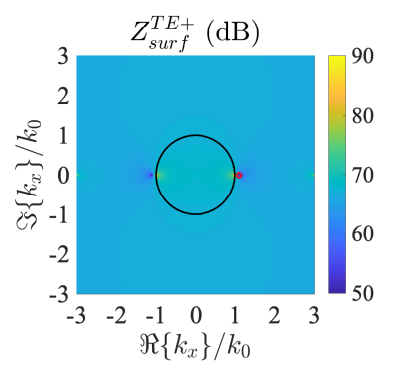

(b)



(c)

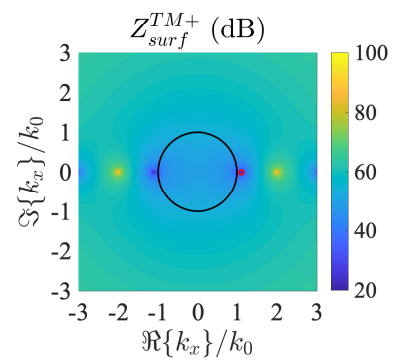

(d)

Fig. 3: Characteristic equation for (a),(b) TE and (c),(d) TM modes at the frequency $f_{\max }$. The plots are shown in dB, thus the poles are illustrated with dark blue spots. The black circle indicate the visible range $\left(\left|k_{x}\right|=k\right)$ and red dot markers represent the calculated poles using [13]. The PPW is air-filled. Parameter settings: $h=\lambda_{\min } / 2$ and $f_{\max }=3 f_{c o}^{T M}$.

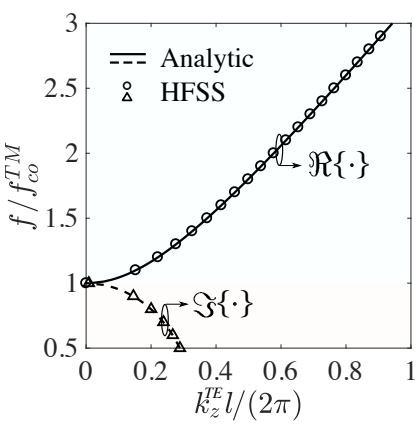

(a) TE modes.

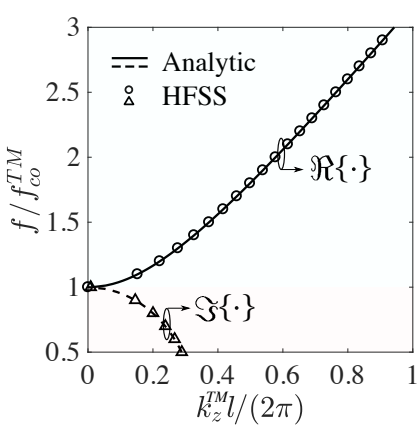

(b) TM modes.
Fig. 4: Dispersion diagram of (a) TE and (b) TM modes for a ED PPW. Parameter settings: $h=\lambda_{\min } / 2, f_{\max }=3 f_{c o}^{T M}$ and $l=\lambda_{\min }$. The PPW is air-filled.

where $\omega_{c o}^{T M}=2 \pi f_{c o}^{T M}$ and $k=k_{0} \sqrt{\epsilon_{r} \mu_{r}}$. When $h \leq$ $\lambda_{\min } / 2=0.5 v / f_{\max }\left(v=1 / \sqrt{\epsilon_{0} \epsilon_{r} \mu_{0} \mu_{r}}\right.$ and $f_{\max }$ is the largest frequency), the cut-off frequency $f_{c o}^{T M}$ can be chosen as $f_{c o}^{T M} \in\left[0, f_{\max }\right]$. As representative example, let us consider an air-filled PPW of height $h=\lambda_{\min } / 2$ and consider $f_{\max }=3 f_{c o}^{T M}$. The CEs (4) and (8) are plotted in $\mathrm{dB}$ in Fig. 3 at the frequency $f_{\max }$. Equations (11) and (12) were used as surface impedance with $L^{T E}$ and $C^{T M}$ being calculated using (13) and (14), respectively. The poles (blue spots in Fig. 3) are equally located in the spectral domain for TE and TM modes, respectively. This is a consequence of having enforced the $\mathrm{ED}$ condition (13). Furthermore, Figs. 3(a) and (c) show that two orthgonally-polarized modes are in propagation $\left(\left|k_{x}\right|<k_{0}\right)$. The corresponding dispersion diagrams of TE and TM modes are shown in Figs. 4(a) and (b), respectively. Fig. 4 shows that two orthogonally-oriented modes are in propagation in the frequency range $\left[f_{c o}^{T M}, f_{\max }\right]$ and their dispersion curves are equal to each other. The length of the considered PPW is $l=\lambda_{\text {min }}$. The analytic formulation, presented in Section II, has been also validated with a full-wave commercial software [15], showing an excellent agreement.

\section{IV. $90^{\circ}$-Phase Delayed Line}

The aim of this Section is to engineer a ED PPW behaving as anisotropic crystal [16]. Two ortho-modes are wanted to be $90^{\circ}$-phased one another, while maintaining their amplitude equal. By setting the cut-off frequency as (14), the eigenvalues $k_{x}^{T M-}$ are found to be constant in frequency. This is clearly visible by equaling equations (12) and (8), and observing that the frequency dependency is deleted when solving for $k_{x}^{T M-}$. Setting the desired phase shift to $\Delta \phi=\pi / 2$, the eigenvalue $k_{x}^{T E-}$ is calculated as a function of $k_{x}^{T M-}$, thus yielding to

$$
k_{x}^{T E-}=\frac{1}{l} \sqrt{\left(k_{x}^{T M-} l\right)^{2}+\Delta \phi\left(-\Delta \phi+2 l k_{z}^{T M-}\right)}
$$

where $l$ is the length of the PPW [see Fig. 1]. When the PPW behaves as $90^{\circ}$-phased line, the ED condition becomes

$$
L_{\Delta \phi}^{T E}=C^{T M} \frac{k_{x}^{T M-}}{k_{x}^{T E-}} \eta^{2} \tan \left(\frac{k_{x}^{T M-} h}{2}\right) \cot \left(\frac{k_{x}^{T E-} h}{2}\right)
$$

where $C^{T M}$ and $k_{x}^{T E-}$ are given by (14) and (15), respectively. Condition (16) shows that $L_{\Delta \phi}^{T E}$ is frequency-dependent. Since $k_{x}^{T M-}$ is constant in frequency, the term responsible for the frequency dependency of $L_{\Delta \phi}^{T E}$ is $k_{x}^{T E-}$. Using (15), it can be observed that $k_{x}^{T E-} \stackrel{l \rightarrow+\infty}{\longrightarrow} k_{x}^{T M-}=$ const. The use of electrically-large PPW's lengths along $z$-axis, i.e., $l \gg \lambda_{\min }$, consequently provides that $L_{\Delta \phi}^{T E}$ becomes a slowly-varying function with frequency and can be so approximated by its mean, thus yielding to

$$
\overline{L_{\Delta \phi}^{T E}}=\frac{1}{\operatorname{meas}\{\Delta f\}} \int_{\Delta f} L_{\Delta \phi}^{T E} d f
$$

where $\Delta f \equiv\left[f_{\max }, f_{c o}^{T M}\right]$.

As example, a $90^{\circ}$-delayed line made of a ED PPW was engineered. The cut-off frequency is $f_{c o}^{T M}=20 \mathrm{GHz}$, thus $C^{T M}$ is calculated as (14). The maximum frequency $f_{\max }$ is twice $f_{c o}^{T M}$ and $h=\lambda_{\min } / 2$. Fig. 5(a) shows the dispersion diagram, when the non-approximated condition (16) is used for $Z_{\text {surf }}^{T E}$. It is shown that the phase difference between two ED ortho-modes is $90^{\circ}$ all over the band $\left[f_{c o}^{T M}, f_{\max }\right]$, when $l=3 \lambda_{\min }$. However, the corresponding $L_{\Delta \phi}^{T E}$ is frequencydependent as plotted in Fig. 5(c). This problem can be solved using its approximated form (17) [see square marker in Fig. $5(\mathrm{c})$ ], whose corresponding dispersion diagram is reported in Fig. 5(b). The use of (17) results in having two quasi-ED ortho-modes in propagation. Their phase difference equals $90^{\circ}$ at only one frequency within the range $\left[f_{c o}^{T M}, f_{\max }\right]$. The 


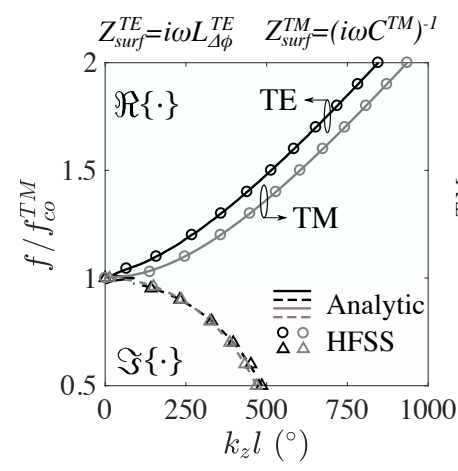

(a)

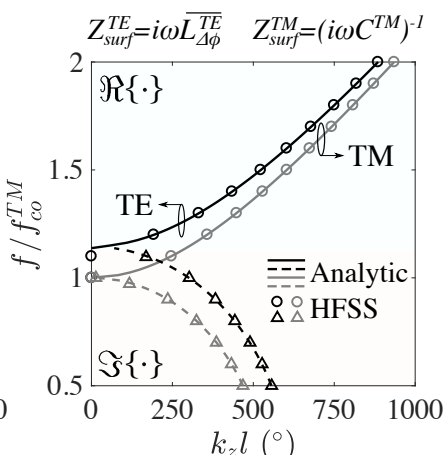

(b)

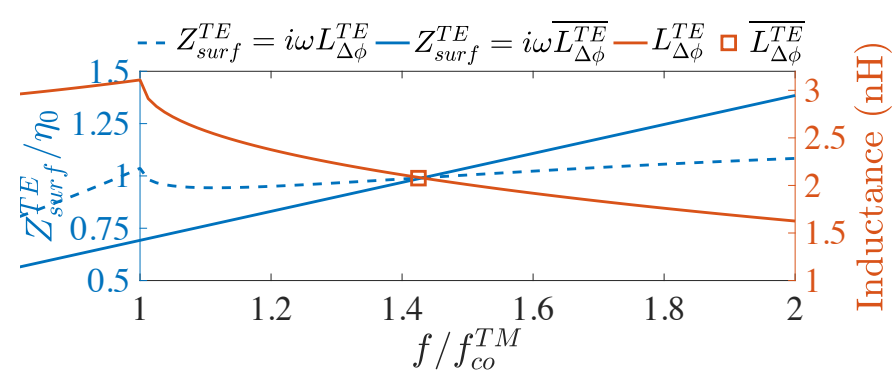

(c)

Fig. 5: Dispersion diagram for (a) $Z_{\text {surf }}^{T E}=i \omega L_{\Delta \phi}^{T E}$ and (b) $Z_{\text {surf }}^{T E}=i \omega \overline{L_{\Delta \phi}^{T E}}$. (c) Surface impedance $Z_{\text {surf }}^{T E}$. Parameter settings: $f_{c o}^{T M}=20 \mathrm{GHz}, h=\lambda_{\min } / 2, f_{\max }=2 f_{c o}^{T M}$ and $l=3 \lambda_{\min }$. The PPW is air-filled.

analytic calculation was also validated using the full-wave simulator [15].

\section{Quasi Equi-dispersive Open-Ended Stubs}

As a difference with [5], open-ended ED PPWs radiate two orthogonally-oriented modes, whose frequency dispersion is the same. The unit cell of the CTS array is shown in Figs. 6(a) and (b) for TM and TE modes, respectively. Periodic boundary conditions are enforced on lateral faces along $x$-axis. Also, perfect magnetic (PMC) and electric (PEC) boundary conditions are alternately considered along $y$-axis for optimal operation of TM and TE modes, respectively. The long-slot array is designed to operate at Ka-band. The width of the slot is $a=\lambda_{\min } / 2$, where $\lambda_{\min }$ is the wavelength at $35 \mathrm{GHz}$. The array periodicity is $d=1.1 a$. The surface IBCs differ for TM and TE mode scenarios and are calculated using (12) and (11), respectively. The capacitance $C^{T M}$ is given by (13), where $f_{c o}^{T M}=20 \mathrm{GHz}$. Also, $\overline{L_{\Delta \phi}^{T E}}$ is calculated using (17). The length of the open-ended stub is $l=5 \lambda_{\min }$ [see Figs. 6(a) and (b)]. The phase of TM and TE modes is plotted in Fig. 6(c) at $29 \mathrm{GHz}$. The two modes are equally-phased at the input section of the stub $(z=0)$. As shown in Fig. 6(c), the aperture exhibits orthogonally-polarized modes that are $90^{\circ}$-phased to each other. The axial ratio (AR) in Fig. 6(d) is computed both analytically and by post-processing the radiated fields using [15]. The analytic calculation was carried out using the numerical model outlined in [5] and is in excellent agreement with full-wave simulations. The AR is

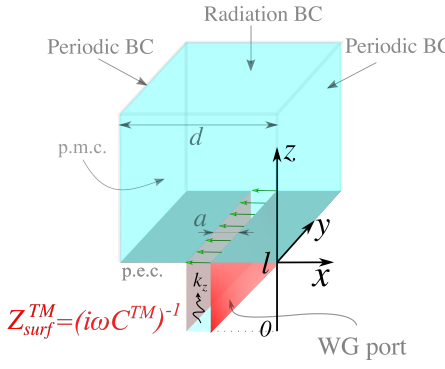

(a) TM mode.

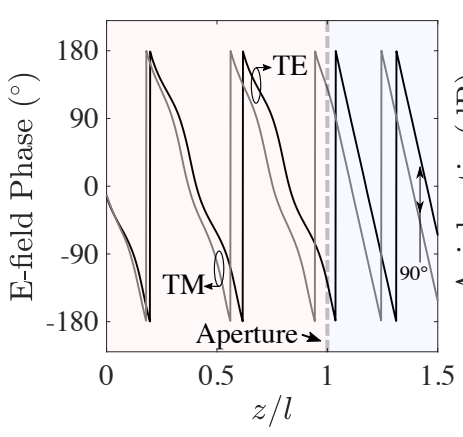

(c)

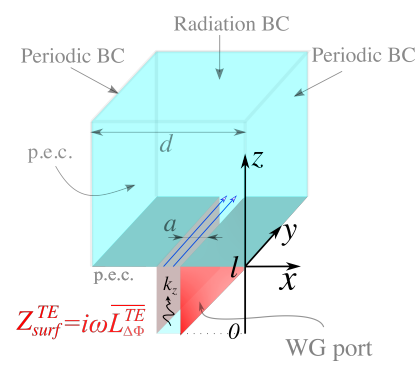

(b) TE mode

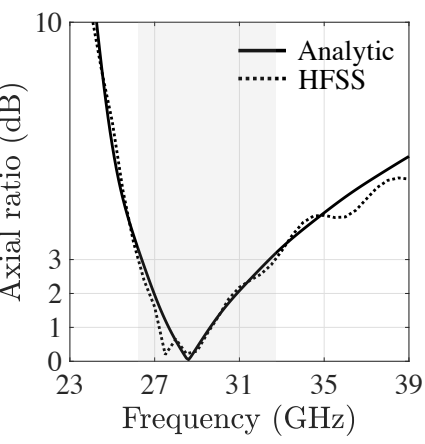

(d)
Fig. 6: Unit cell of the CTS array for (a) TM and (b) TE mode operation. (c) Phase difference as function of the variable $z$ at $29 \mathrm{GHz}$. (d) Axial ratio versus frequency. The width of the slots is $a=4.28 \mathrm{~mm}$ and the array periodicity is $d=1.1 a$.

below $3 \mathrm{~dB}$ over the band $26.8-32.2 \mathrm{GHz}$ (22.3\% of relative bandwidth) for broadside radiation.

A concrete way to realize the antenna feeding might involve the use of quasi-optical systems, such as [19]. Furthermore, physical structures, that may realize reactive sheets, rely on metallic patterns printed on grounded dielectric slabs, as done in [17], [18]. These structures resemble anisotropic metawaveguides and can be designed by following the approach outlined in [20]-[22]. Basically, independent circuit models are employed to reconstruct the desired surface reactance, according to the E-field polarization. Since surface impedances are here demanded to be inductive and capacitive for $y$ - and $x$ polarized E-fields, respectively, their physical realization can be approached separately. Owing to their geometrical shape, meandered Jerusalem crosses [20], [21] may represent a valid candidate as meta-waveguide's unit cell. Their design can be carried out by following the approach used in [22].

\section{CONCLUSION}

The modal analysis of paralel plate waveguides with purely reactive walls has been reported in this letter. This class of waveguides can support the propagation of two equidispersive, orthogonally-polarized modes. The engineering of boundary conditions was provided through analytic closedform expressions. This type of waveguide can be employed as open-ended radiating stubs. A representative example in Kaband shows that a fractional $3-\mathrm{dB}-\mathrm{AR}$ bandwidth of $22.3 \%$ is achieved for broadside radiation. 


\section{REFERENCES}

[1] M. Chen and G. Tsandoulas, "A wide-band square-waveguide array polarizer," Trans. Antennas Propag., vol. 21, no. 3, pp. 389-391, May 1973.

[2] J. Bornemann and V. A. Labay, "Ridge waveguide polarizer with finite and stepped-thickness septum," IEEE Trans. Microw. Theory Tech., vol. 43, no. 8, pp. 1782-1787, Aug. 1995.

[3] G. Tsandoulas and G. Knittel, "The analysis and design of dualpolarization square-waveguide phased arrays," Trans. Antennas Propag., vol. 21, no. 6, pp. 796-808, November 1973.

[4] N. Bartolomei, M. Garcia-Vigueras, F. Doucet, D. Blanco, E. Girard, R. Sauleau, and M. Ettorre, "Circularly polarized parallel plate waveguide multiple-beam lens-like antenna for Satcom applications," 13th Eur. Conf. Antennas Propag. (EUCAP), Krakow, Poland, 2019, pp. 1-3.

[5] M. Del Mastro, F. Foglia Manzillo, M. Śmierzchalski, D. GonzlezOvejero, P. Pouliguen, P. Potier, R. Sauleau, and M. Ettorre, ”Analysis of circularly polarized CTS arrays," Trans. Antennas Propag., vol. 68, no. 6, pp. 4571-4582, June 2020, doi: 10.1109/TAP.2020.2972438.

[6] M. Del Mastro, F. Foglia Manzillo, M. Śmierzchalski, D. GonzlezOvejero, P. Pouliguen, P. Potier, R. Sauleau, and M. Ettorre, "Circularlypolarized CTS arrays," 13th Eur. Conf. Antennas Propag. (EUCAP), Krakow, Poland, 2019, pp. 1-3.

[7] M. Smierzchalski, F. Foglia Manzillo, M. Del Mastro, N. Capet, B. Palacin, R. Sauleau, and M. Ettorre, "A novel dual-polarized continuous transverse stub antenna based on corrugated waveguides - part I: principle of operation and design," Trans. Antennas Propag., 2020.

[8] M. Smierzchalski, F. Foglia Manzillo, M. Del Mastro, N. Capet, B. Palacin, R. Sauleau, and M. Ettorre, "A novel dual-polarized continuous transverse stub antenna based on corrugated waveguides - part II: experimental demonstration," Trans. Antennas Propag., 2020.

[9] N. Marcuvitz, "Waveguide handbook," McGraw-Hill, 1951.

[10] D. M. Pozar, "Microwave engineering - 3th ed.," John Wiley \& Sons, 2005.

[11] L. B. Felsen, and N. Marcuvitz, "Radiation and scattering of waves," Hoboken, NJ, USA: Wiley/IEEE Press, 1994.

[12] J. R. Wait, "On the theory of shielded surface waves," IEEE Trans. Microw. Theory Tech., vol. 15, no. 7, pp. 410-414, July 1967.

[13] V. Galdi, and I. M. Pinto, "Simple algorithm for accurate location of leaky-wave poles for grounded inhomogeneous dielectric slabs," Microwave and Optical Technology Letters, vol. 24, no. 2, pp. 135-140, 2000.

[14] R. B. Dybdal, L. Peters, and W. H. Peake, "Rectangular waveguides with impedance walls," IEEE Trans. Microw. Theory Tech., vol. 19, no. 1, pp. 2-8, Jan. 1971.

[15] ANSYS Electronics Desktop 2018.2, HFSS High-frequency structure simulation, 2018.

[16] M. Born, and E. Wolf, "Principles of optics, 7th ed." Cambridge University Press, 1999.

[17] M. N. M. Kehn, M. Nannetti, A. Cucini, S. Maci and P. -S. Kildal, "Analysis of dispersion in dipole-FSS loaded hard rectangular waveguide," Trans. Antennas Propag., vol. 54, no. 8, pp. 2275-2282, Aug. 2006, doi: 10.1109/TAP.2006.879198.

[18] O. Luukkonen et al., "Simple and accurate analytical model of planar grids and high-impedance surfaces comprising metal strips or patches," Trans. Antennas Propag., vol. 56, no. 6, pp. 1624-1632, June 2008, doi: 10.1109/TAP.2008.923327.

[19] M. Ettorre, R. Sauleau and L. Le Coq, "Multi-beam multi-layer leakywave SIW pillbox antenna for millimeter-wave applications," Trans. Antennas Propag., vol. 59, no. 4, pp. 1093-1100, April 2011, doi: 10.1109/TAP.2011.2109695.

[20] M. Del Mastro, M. Ettorre and A. Grbic, "Dual-band, orthogonallypolarized LP-to-CP converter for SatCom applications," Trans. Antennas Propag., doi: 10.1109/TAP.2020.2989868.

[21] M. D. Mastro, M. Ettorre and A. Grbic, "Analytic Design Of DualBand, Dual-Polarized Lp-to-Cp Polarization Converters," 14th Eur. Conf. Antennas Propag. (EUCAP), Copenhagen, Denmark, 2020, pp. 1-3, doi: 10.23919/EuCAP48036.2020.9135304.

[22] M. Ettorre, S. M. Rudolph and A. Grbic, "Generation of propagating Bessel beams using leaky-wave modes: Experimental Validation," Trans. Antennas Propag., vol. 60, no. 6, pp. 2645-2653, June 2012, doi: 10.1109/TAP.2012.2194674. 